\title{
Open issues in antibody-mediated encephalitis
}

\author{
Luigi Zuliani*,1,2 (D) \& Marco Zoccarato 2,3 \\ ${ }^{1}$ Department of Neurology, San Bortolo Hospital, AULSS8 Berica, Vicenza, Italy \\ ${ }^{2}$ Neuroimmunology Group, Pediatric Research Institute "Città della Speranza", Padova, Italy \\ ${ }^{3}$ Department of Neurology, Sant'Antonio Hospital, Padua, Italy \\ *Author for correspondence: Tel.: +39393771910; luigi.zuliani@aulss8.veneto.it
}

First draft submitted: 2 December 2019; Accepted for publication: 16 December 2019; Published online: 11 March 2020

Keywords: AK5 • autoimmune encephalitis • immune-checkpoint inhibitors • LGI1 - IgLON5 • limbic encephalitis • NMDAR • NSAb • NSAE

The field of autoimmune encephalitis (AE) associated with antibodies against targets located on the surface of neurons (i.e., neuronal surface antibodies [NSAbs]-associated encephalitis [NSAE]) has grown immensely in the last 10 years. This growth has been in terms of both published research of novel antibodies and pathogenic mechanisms, and the awareness and knowledge among neurologists and clinicians, although much remains to be done for those who are not neurologists [1].

Together with the expanding knowledge about other inflammatory autoimmune diseases such as neuromyelitis optica spectrum disorders, growing interest in NSAE has led to the emergent field of 'autoimmune neurology', a branch of neuro-immunology focusing on autoimmune, usually antibody-associated, diseases of the central nervous system other than multiple sclerosis [2].

The discovery of a number of antibodies targeting synaptic proteins has completely changed the approach to neurologic and psychiatric disorders that were previously considered idiopathic or not immune mediated [3]. Other possible novel antibodies are still to be discovered and known syndromes are to be fully characterized.

In order to better manage this new field of neurology, for which specific laboratory and clinical skills are necessary, we and other experts promoted a national collaboration network aimed at data collection and standardization of procedures and management [4].

\section{Diagnosis}

NSAEs are rare diseases with a probable annual incidence of one to five cases per million of the population [5]. The real incidence is unknown. Adequate epidemiological studies are lacking, first because these disorders have been characterized only very recently and secondly, because these syndromes are probably still under recognized [6].

Although knowledge about NSAEs has greatly increased among adult and child neurologists and an international panel has proposed guidelines for the clinical diagnosis of AE [6], the diagnosis of NSAE is still a major issue.

\section{Laboratory diagnostics}

Diagnosis of NSAbs is challenging for several reasons, including the heterogeneous availability of NSAb testing among different hospitals and countries, and the rapidly increasing number of novel NSAbs, for which testing is available in reference centers only.

In Italy, for instance, commercial assays to detect NSAbs are commonly available in almost every hospital but laboratories with real clinical and technical expertise in this field are few. While the experts recommend the use of indirect immunohistochemistry (IHC)/immunofluorescence on tissues or neurons as an initial test to screen for the presence of autoantibodies, in routine laboratory practice, commercial assays are the first and often the only test that is performed. Considering this framework, the Italian Association of Neuroimmunology experts panel proposed that after a first level approach with diffusely available commercial assays, in-house indirect immunohistochemistry/immunofluorescence should be performed in reference laboratories. Also, that both the phenotypes and laboratory results of clinically suggestive cases should be discussed with neurologists with clinical 
and technical expertise $[7,8]$. Failure to apply this approach may lead to under-diagnosis or potentially incorrect diagnosis, accompanied by useless and harmful therapies. Given the lack of validated diagnostic gold standards and considering that there are no biomarkers other than antibodies, diagnosis of both NSAE and paraneoplastic neurological syndromes demands great expertise.

\section{Differential diagnosis}

The diagnostic approach to patients, who are subacutely developing symptoms resembling NSAE, such as memory deficits, psychiatric features and seizures, should consider challenging clinical overlaps. For example, NSAE with prominent cognitive involvement can be misdiagnosed with prion disease, like Creutzfeldt-Jakob disease, or other rapid forms of neurodegenerative dementia [9]. The recent description of IgLON5 antibodies in patients with movement and sleep disorders [10] and of AK5 antibodies in patients with prominent memory deficits and lack of epileptic features [11] (for which commercial tests are not yet available [12]), further underlines the importance of searching for NSAbs when faced with an atypical presentation of suspected neurodegenerative etiology.

Neuroimaging plays a crucial role in the differential diagnosis of AE. While brain MRI in anti-NMDAR encephalitis, which is probably the most frequent form of NSAE, is often negative, approximately $70 \%$ of autoimmune limbic encephalitis (LE) demonstrates a hyperintense signal in MRI T2-weighted images of mesial temporal structures. Notably, similar MRI abnormalities can be demonstrated in infectious diseases and tumors. Indeed, radiological differentiation between autoimmune LE and mesial temporal glioma is not an uncommon issue in clinical practice and some clues may help to distinguish these patterns, as is previously suggested [13].

\section{Triggers}

Systemic tumors and viral infections, especially herpetic infection, are well-recognized triggers of NSAE, for example, anti-NMDAR encephalitis after herpetic encephalitis [14]. Accumulating data suggest that also immune system dysregulation generated by immunologic therapy can determine AE. Post-transplant immunosuppression [15] and monoclonal antibodies like alemtuzumab [16], have been associated with the development of AE. Immune checkpoint inhibitors (ICIs) are drugs that are increasingly used in oncologic therapy, with the aim to enhance T-cell response against tumoral cells. A wide number of autoimmune conditions have been associated with the use of ICIs, involving the central and peripheral nervous systems [17]. Some of these syndromes fulfill diagnostic criteria for NSAE or for paraneoplastic neurological syndromes and are associated with antibodies [18]. The real incidence of NSAE in patients treated with ICIs should be assessed in prospective studies.

\section{Nomenclature \& categorization}

The growing interest in NSAE has led in recent years, to recognition of an autoimmune etiology in syndromes with isolated or prominent clinical features. Thus, new categories, such as 'autoimmune epilepsy', 'autoimmune dementia' and 'autoimmune movement disorders', have increasingly appeared in the literature and at international meetings.

Lately, the concept of psychiatric disturbances of an autoimmune origin has also strongly emerged, arousing great debate among experts on the lack of studies focusing on the presence of NSAbs in the CSF, which have higher diagnostic relevance. The recently published position paper on 'autoimmune psychosis' attempted to bring some clarity [19]. Pollak et al., sought to rigorously provide some clues (red flags) to prompt a clinician to suspect a possible autoimmune etiology. Clinical neuro-immunologists (or autoimmune neurologists) tend to consider this categorization with some skepticism. The idea of postulating novel autoimmune syndromes starting from the isolation of single clinical features, although prominent, may appear artificial or at least short-sighted as, eventually, these are forme fruste of AE. However, it must be acknowledged that this kind of categorization can be useful from an operational point of view, in that they increase awareness and the rate of diagnosis of potentially reversible disorders.

\section{Management}

Irrespective of how NSAE are diagnosed or classified, once the diagnosis of NSAE is established, the choice of the best treatment option and its timing remains the most important open issue. The therapeutic approach is based on retrospective studies and expert opinion. Clinical trials are lacking or are very difficult to be completed as recently demonstrated by the randomized controlled trial designed by Dubey et al., with IVIG in LGI1/CASPR2 antibodies autoimmune epilepsy [20]. 
To address the challenges of NSAE diagnosis and treatment, the Italian Working Group on Autoimmune Encephalitis has developed a consensus document [4]. The advice provided is addressed to the clinician faced with a patient with NSAE, who must manage different issues in different phases of the disease. Thus, in the early phase (24-72 h after hospitalization), the first aim is to rule out other differential diagnoses with strong therapeutic implications, for example, infectious encephalitis. In the later phase (the following days-weeks), the aim is to refine the diagnosis and start or continue adequate immunotherapy without delay, escalating from 'first line', such as steroids, IVIG, plasma-exchange to 'second line' therapies, including immunosuppressant drugs, as anti-CD20 antibodies or alkylating agents.

Another major issue is represented by antibody-negative cases, considering that in world reference laboratories at least $7 \%$ of clinically definite LE are antibody negative [21]. Although recently published guidelines by Graus et al., addressed the problem of providing criteria to diagnose possible and probable AE, independently of antibody results, it is not surprising that antibody-negative AE, which are mainly diagnosed after exclusion of other disorders, tend to have a poorer prognosis because of delayed treatments, as demonstrated in a recent Italian multicenter retrospective study [22].

In both antibody-positive and -negative $\mathrm{AE}$, the most effective treatment combinations, long-term management and clinical and paraclinical follow-up are major issues for which prospective studies are necessary.

\section{Conclusion}

In conclusion, NSAE are rare and still difficult to diagnose disorders. The diversity of neurologic presentations and the peculiar immunopathogenic mechanism imply knowledge of laboratory diagnostics and of different neurological disciplines.

Given the rarity and heterogeneity of these disorders, it seems unlikely that multicenter randomized clinical trials could inform the clinical practice of NSAE in the coming years. Meanwhile, panels of experts, possibly international neurological society task forces, are warranted to address these issues.

\section{Acknowledgments}

Writing assistance was utilized in the production of this manuscript. The authors thank J Fleming for reviewing the manuscript.

\section{Financial \& competing interests' disclosure}

The authors have no other relevant affiliations or financial involvement with any organization or entity with a financial interest in or financial conflict with the subject matter or materials discussed in the manuscript apart from those disclosed.

No writing assistance was utilized in the production of this manuscript.

\section{Open access}

This work is licensed under the Attribution-NonCommercial-NoDerivatives 4.0 Unported License. To view a copy of this license, visit http://creativecommons.org/licenses/by-nc-nd/4.0/

\section{References}

1. Dalmau J, Graus F. Antibody-mediated encephalitis. N. Engl. J. Med. 378, 840-851 (2018).

2. López-Chiriboga AS, Clardy SL. Emerging subspecialties in neurology: autoimmune neurology. Neurology 89, e129-e133 (2017).

3. Dalmau J. NMDA receptor encephalitis and other antibody-mediated disorders of the synapse. Neurology 87, 2471-2482 (2016).

4. Zuliani L, Nosadini M, Gastaldi M et al. Management of antibody-mediated autoimmune encephalitis in adults and children: literature review and consensus-based practical recommendations. Neurol Sci. 40, 2017-2030 (2019).

5. Dubey AD, Pittock SJ, Kelly CR et al. Autoimmune Encephalitis Epidemiology and a comparison to infectious encephalitis. Ann. Neurol. 83, 166-177 (2018).

6. Graus F, Titulaer MJ, Balu R et al. A clinical approach to diagnosis of autoimmune encephalitis. Lancet Neurol. 15, 391-404 (2016).

7. Zuliani L, Zoccarato M, Gastaldi M et al. Diagnostics of autoimmune encephalitis associated with antibodies against neuronal surface antigens. Neurol Sci. 38, 225-229 (2017).

8. Zoccarato M, Gastaldi M, Zuliani L et al. Diagnostics of paraneoplastic neurological syndromes. Neurol. Sci. 38, 237-242 (2017).

9. Geschwind MD, Shu H, Haman A et al. Rapidly progressive dementia. Ann. Neurol. 64, 97-108 (2008).

10. Sabater L, Gaig C, Gelpi E et al. A novel non-rapid-eye movement and rapid-eye-movement parasomnia with sleep breathing disorder associated with antibodies to IgLON5: a case series, characterisation of the antigen, and post-mortem study. Lancet Neurol. 13, 575-586 (2014). 
11. Tüzün E, Rossi JE, Karner SF et al. Adenylate kinase 5 autoimmunity in treatment refractory limbic encephalitis. J. Neuroimmunol. 186, 177-180 (2007).

12. Bien CI, Nehls F, Kollmar R et al. Identification of adenylate kinase 5 antibodies during routine diagnostics in a tissue-based assay: three new cases and a review of the literature. J. Neuroimmunol. 334, 1-6 (2019).

13. Zoccarato M, Valeggia S, Zuliani L et al. Conventional brain MRI features distinguishing limbic encephalitis from mesial temporal glioma. Neuroradiology 61(8), 853-860 (2019).

14. Armangue T, Spatola M, Vlagea A et al. Frequency, symptoms, risk factors, and outcomes of autoimmune encephalitis after herpes simplex encephalitis: a prospective observational study and retrospective analysis. Lancet Neurol. 17, 760-772 (2018).

15. Cohen DA, Sebastian Lopez-Chiriboga A, Pittock SJ et al. Posttransplant autoimmune encephalitis. Neurol. Neuroimmunol .NeuroInflamm. 5, 4-6 (2018).

16. Giarola B, Massey J, Barnett Y et al. Autoimmune encephalitis following alemtuzumab treatment of multiple sclerosis. Mult. Scler. Relat. Disord. 28, 31-33 (2019).

17. Yshii LM, Hohlfeld R, Liblau RS. Inflammatory CNS disease caused by immune checkpoint inhibitors: status and perspectives. Nat. Rev. Neurol. 13, 755-763 (2017).

18. Graus F, Dalmau J. Paraneoplastic neurological syndromes in the era of immune-checkpoint inhibitors. Nat. Rev. Clin. Oncol. Mar. 16(9), 535-548 (2019).

19. Pollak TA, Lennox BR, Müller $S$ et al. Autoimmune psychosis: an international consensus on an approach to the diagnosis and management of psychosis of suspected autoimmune origin. Lancet Neurol. S2215-0366(19), 30290-30291 (2019).

20. Dubey D, Britton J, Mckeon A et al. Randomized placebo controlled trial of IVIG in autoimmune LGI1/CASPR2 epilepsy. Ann. Neurol.87(2), 313-323 (2019).

21. Graus F, Escudero D, Oleaga L et al. Syndrome and outcome of antibody-negative limbic encephalitis. Eur. J. Neurol. 25, 1011-1016 (2018).

22. Gastaldi M, Mariotto S, Giannoccaro MP et al. Subgroup comparison according to clinical phenotype and serostatus in autoimmune encephalitis: a multicenter retrospective study. Eur. J. Neurol.27(4), 633-643 (2019). 